\title{
Torsión testicular en testes no descendidos
}

\author{
Domínguez Hinarejos C, Vivancos Garbayo S, Bonillo García MA, Serrano Durbá A, \\ García Ibarra F.
}

Departamento de Cirugía Pediátrica. Sección de Urología Infantil. Hospital Infantil La Fe. Valencia.

Actas Urol Esp. 2007;31(1):49-51

\section{RESUMEN}

\section{TORSIÓN TESTICULAR EN TESTES NO DESCENDIDOS}

La torsión del teste criptorquídico presenta una sintomatología inespecífica, cuya sospecha clínica nos obliga a una exploración quirúrgica, ya que a pesar de considerar a la ecografia Doppler-color la prueba de imagen recomendada, sus criterios de isquemia testicular son discutidos por su porcentaje nada desdeñable de falsos negativos. En cuanto al tratamiento, se realizará una orquiectomía ante una clara necrosis testicular siendo controvertida la actitud terapéutica del testículo contralateral. Nuestra filosofia es la no fijación sistemática del teste solitario porque pensamos que es más importante la educación sanitaria al respecto de los padres y posteriormente del adolescente, para así conseguir un diagnóstico precoz de una futura torsión.

Palabras Clave: Torsión testicular. Teste no descendido. Criptorquidia.

\section{ABSTRACT \\ TORSION OF UNDESCENDED TESTIS}

Torsion of a cryptorchidid testicle presents a non-specific symptomatology. Clinical suspicion indicates surgical exploration, irrespective of Doppler ultrasound with its inherent false negative results. With regards to treatment, an orchiectomy is performed when the testis is necrotic. Management of the contralateral testis is controversial. Our policy is not to fix them systematically because we believe that a healthy education of parents and children is more important, so as to obtain an earlier diagnosis of a future torsion.

Key words: Testicular torsion. Undescended testis. Criptorchidia.

$\mathrm{E}^{1}$ primer caso de torsión testicular afectando a un teste criptorquídico fue descrito por Dalasiavue en $1840^{1}$. En la literatura revisada son pocos los casos descritos y su etiopatogenia no es del todo conocida. La mala o nula fijación de estos testículos a la bolsa escrotal, junto a probables contracciones espasmódicas del músculo cremáster ${ }^{2}$, pueden influir en la susceptibilidad de estos testes a ser torsionados.

A continuación presentamos nuestra experiencia en esta patología exponiendo tres casos clínicos con el fin de resaltar la importancia de un diagnóstico precoz.

\section{CASOS CLÍNICOS}

Durante los años 1995-2001 se han registrado 3 casos de testes criptorquídicos torsionados a diferentes edades: 30 días, 9 meses y 13 años. Su clínica fue variada, presentándose en los 2 niños de menor edad en forma de llanto incoercible acompañado de inquietud e intolerancia a las tomas, diagnosticados en su centro de salud de un dolor abdominal inespecífico dándose de alta al paciente con tratamiento conservador. A pesar de ello, los niños no evolucionaron bien por lo que los remiten al servicio de urgencias de nuestro centro. El otro varón de 13 años acudió por 
dolor inguinal de comienzo súbito asociado a náuseas. La exploración física (EF) fue similar en los tres pacientes se apreció un edema y tumoración a nivel inguinal con signos inflamatorios, irreductible, dolorosa a la palpación y hemiescroto ipsilateral vacío.

La ecografia-doppler realizada en 2 de los casos (neonato y prepuber) mostró una disminución o nula vascularización testicular, aumentado de tamaño y ecogenicidad heterogénea. El otro, de 9 meses de edad, no demostró alteraciones significativas del flujo arterial, pero en el acto quirúrgico sí se objetivó una necrosis de la gónada.

En todos ellos se practicó una exploración quirúrgica bajo anestesia general evidenciándose 2 testes necróticos por torsión del cordón espermático tras 24 horas y 5 días de evolución respectivamente, por lo que se llevó a cabo la orquiectomía. Por el contrario, el niño de 13 años, que presentaba una torsión de cordón de 180ㅜㅡ, al detorsionarlo manualmente y aplicarle calor local, recuperó su coloración parcialmente por lo que se le practicó una orquidopexia según la técnica de Schumaker presentando actualmente un teste viable después de 1 año de evolución.

\section{DISCUSIÓN}

La torsión del teste criptorquídico es más frecuente en el adulto joven ${ }^{2}$ aunque se han descrito varios casos en la literatura afectando al recién nacido ${ }^{3}$. Si el diagnóstico de torsión testicular en su localización escrotal resulta difícil, en el teste criptorquídico la dificultad es mayor por dos motivos: porque no se piensa en ella y porque las exploraciones complementarias son menos sensibles cuando el teste está ubicado fuera del escroto. Por ello ante un niño, independientemente de su edad, con sintomatología abdominal no específica y escroto vacío es necesario pensar en esta patología.

El diagnóstico diferencial que se nos plantea ante un niño con llanto y una exploración que muestre una masa inguinal irreductible con un escroto vacío ipsilateral prácticamente se reducen a descartar una hernia inguinal incarcerada, un tumor abdominal o testicular y la torsión de un testículo criptorquídico ${ }^{4}$. Por lo tanto, se deberá realizar una exploración quirúrgica con doble finalidad, diagnóstica y terapéutica.
La viabilidad del testículo va ligada directamente a un diagnóstico y a un tratamiento precoz y ese debe ser nuestro objetivo. En nuestro caso, los niños de 30 días y nueve meses presentaron una clínica tan larvada e inespecífica que hizo retrasar su diagnóstico, lo que condujo a una necrosis testicular. Por el contrario gracias a la mejor expresividad clínica que presentó el niño de 13 años se logró sospechar precozmente esta patología y por tanto no diferir la cirugía posibilitando conservar el testículo.

De todos los métodos diagnósticos que podamos emplear en la actualidad se puede considerar que el Doppler-color ha desplazado a la ecografía convencional y a los estudios isotópicos con Tecnecio. Aún así los criterios de isquemia testicular del eco-Doppler son discutidos por su porcentaje nada desdeñable de falsos negativos ${ }^{5}$.

Varios autores ${ }^{5,6}$ han comprobado que la presencia de flujo vascular en un estudio de este tipo no nos excluye la torsión testicular tal y como ocurrió en uno de nuestros pacientes ya que una correcta valoración de la perfusión vascular dependerá del grado de torsión y del tiempo de evolución.

Kravchick $^{6}$ y colaboradores obtuvieron una sensibilidad del $77,8 \%$ y una especificidad del 90\% con el empleo del Doppler-color en 38 pacientes con alta sospecha de torsión testicular. Llegan a concluir, al igual que Steinhardt ${ }^{7}$, que el mejor uso de esta prueba diagnóstica sería la confirmación de una impresión clínica: de ausencia de torsión testicular demostrando flujo vascular y de patología escrotal aguda cuando se evidencia un hiperaflujo vascular. Nos debe alertar, sin embargo, un Doppler que objetive una asimetría de vascularización en un teste de ecogenicidad heterogénea ${ }^{8}$.

Con respecto al tratamiento, nuestra actitud es la realización de una orquiectomía ante un clara necrosis testicular cuando no exista recuperación de coloración tras la detorsión y aplicación de calor local durante 15-20 minutos o aparición de líquido hemorrágico intravaginal con afectación testicular importante.

La controversia surge en la actitud terapéutica a adoptar con respecto al testículo contralateral. Varios autores ${ }^{1-4}$ están a favor de la orquidopexia del mismo apoyando la hipótesis que ya 
Lyon estableció en $1961^{9}$ al afirmar que la alteración anatómica que favoreció la torsión puede presentarse bilateralmente. Así Abeshouse ${ }^{10}$ en 1963 confirma que un 5-30\% de los testículos contralaterales no fijados sufrirán también una torsión intravaginal. En contraposición está la actitud de Duckett ${ }^{11}$ respecto al testículo contralateral que será de vigilancia por parte de los padres y de actuación rápida ante la aparición de determinados signos de alarma.

Nuestra filosofia es la no fijación sistemática del teste solitario porque pensamos que es más importante la educación sanitaria al respecto de los padres y posteriormente del adolescente para así conseguir un diagnóstico precoz. Opinamos que se trata de una manipulación innecesaria del testículo, modificando su anatomía y tal vez por ello predisponiéndolo a la torsión ${ }^{12}$. Bellinger ${ }^{13}$ en 1989 demostró experimentalmente en ratas que la sutura empleada en la fijación conducía a un daño local testicular. Además se han registrado varios casos de torsión testicular años después de haberlos pexiados ${ }^{14,15}$.

Por todo ello pensamos que si lo fijamos además de poder producir una alteración en el parénquima testicular (rotura de la barrera hematotesticular, hemorragia e infección) creamos una falsa seguridad por un exceso de confianza al presentar el antecedente de una orquidopexia previa y por tanto no pensar en la posibilidad de torsión testicular.

\section{CONCLUSIONES}

- Ante un niño con dolor abdominal inespecífico y hemiescroto vacío se debe pensar en la posibilidad de torsión de un teste criptorquídico.

- Nuestra actitud es la no fijación sistemática del teste contralateral como profilaxis de una posible torsión, ni siquiera en las monorquias testiculares secundarias a otras patologías, por la falsa seguridad que puede crear en una patología donde el diagnostico precoz es tan necesario.

\section{REFERENCIAS}

1. Mishriki SF, Winkle DC, Frank JD. Fixation of a single testis: always, sometimes or never. Br J Urol.1992;69(3): 311 313.

2. Mowad JJ, Konvolinka CW. Torsion of undescended testis. Urology. 1978;12(5):567-568.

3. Nistal M, Regadera J, Redondo E, Paniagua R. Cystic intraabdominal testicular torsion in an infant. J Urol. 1985; 133(3):481-482.

4. Rabii R, Rais H, Hafiani M, Dassouli BE, Bennani S, el Mrini M, et al. Torsion of an undescended testis. Apropos of a case. Ann Urol. (Paris) 1998;32(1):49-51.

5. Hrebinko RL, Bellinger MF. The limited role of imaging techniques in managing children with undescended testes. J Urol. 1993;150(2 Pt 1):458-460.

6. Kravchick S, Cytron S, Leibovici O, Linov L, London D, Altshuler A, et al. Color Doppler sonography: its real role in the evaluation of children with highly suspected testicular torsion. Eur Radiol. 2001;11(6):1000-1005.

7. Steinhardt GF, Boyarsky S, Mackey R. Testicular torsion: pitfalls of color Doppler sonography. J Urol. 1993;150(2 Pt 1):461-462.

8. Fernández M, Dominguez C. Utilidad del doppler color en el escroto agudo de los niños. Cir Pediatr. 1997;10:25-28.

9. Lyon RP.Torsion of the testicle in childhood. A painless emergency requiring contralateral orchiopexy. Jama 1961; 178:702-705.

10. Abeshouse B. Torsion of the spermatic cord: report of three cases and review of the literature. Urologic Cutaneous review 1963;40:699.

11. Duckett J. Routine contralateral exploration and fixation is unjustified. Dial Pediatr Urol. 1991;14:7.

12. Cooper CS, Snyder OB, Hawtrey CE. Bilateral neonatal testicular torsion. Clin Pediatr. (Phila) 1997;36(11):653656.

13. Bellinger MF, Abromowitz H, Brantley S, Marshall G. Orchiopexy: an experimental study of the effect of surgical technique on testicular histology. J Urol. 1989;142 (2 Pt 2):553-555; discussion 572.

14. O'Shaughnessy M, Walsh TN, Given HF. Testicular torsion following orchidopexy for undescended testis. Br J Surg. 1990;77 (5):583.

15. May RE, Thomas WE. Recurrent torsion of the testis following previous surgical fixation. Br J Surg. 1980;67(2): 129-130.

Correspondencia autor: Dr. C. Domínguez Hinarejos. Sección de Urología Infantil. Hospital Infantil La Fe. Avda. Campanar, 21. 46009 Valencia. Tel.: 963862700

E-mail autor: dominguezhinar@hotmail.com

Información artículo: Nota clínica

(Trabajo aceptado mayo 2006) 